\title{
Approximate Solutions for a Class of Fractional-Order Model of HIV Infection via Linear Programming Problem
}

\author{
Samaneh Soradi Zeid', Mostafa Yousefí2 ${ }^{2}$, Ali Vahidian Kamyad ${ }^{3}$ \\ ${ }^{1}$ Department of Mathematics, Faculty of Mathematics, University of Sistan and Baluchestan, Zahedan, Iran \\ ${ }^{2}$ National Iranian Oil Products Distribution Company (NIOPDC), Zahedan Region, Zahedan, Iran \\ ${ }^{3}$ Department of Mathematics, Ferdowsi University of Mashhad, Mashhad, Iran \\ Email: s_soradi@yahoo.com, mostafayousefi12@gmail.com, avkamyad@yahoo.com
}

Received 14 May 2016; accepted 24 June 2016; published 27 June 2016

Copyright (C) 2016 by authors and Scientific Research Publishing Inc.

This work is licensed under the Creative Commons Attribution International License (CC BY).

http://creativecommons.org/licenses/by/4.0/

c) (i) Open Access

\begin{abstract}
In this paper, we provide a new approach to solve approximately a system of fractional differential equations (FDEs). We extend this approach for approximately solving a fractional-order differential equation model of HIV infection of $\mathrm{CD}^{+} \mathrm{T}$ cells with therapy effect. The fractional derivative in our approach is in the sense of Riemann-Liouville. To solve the problem, we reduce the system of FDE to a discrete optimization problem. By obtaining the optimal solutions of new problem by minimization the total errors, we obtain the approximate solution of the original problem. The numerical solutions obtained from the proposed approach indicate that our approximation is easy to implement and accurate when it is applied to a systems of FDEs.
\end{abstract}

\section{Keywords}

Riemann-Liouville Derivative, Fractional HIV Model, Optimization Linear Programming, Discritezation

\section{Introduction}

In recent years, scientists have been interested in studying the fractional calculus and the FDEs in different fields of engineering, physics, mathematics, biology, finance, biomechanics and electrochemical processes (see [1]-[8], for more details). Also, it has been shown that modelling the behavior of many biological systems that governed by FDEs has more advantages than classical integer-order modelling [9]. Readers interested in FDEs are referred to [10]-[17]. Although great efforts have been made to find numerical and analytical techniques for 
solving FDE, for example, predictor-corrector method [18], the Adomian decomposition [19], the variational iteration method [20], collocation using spline functions [21] and matrix expression given by [22] [23], but most of these FDEs do not have analytic solutions.

In this paper, at first, we approximate the fractional derivative by a finite difference method and then use the AVK approach [24] to obtain a new approximate solution for the FDEs. This approach substitutes the FDEs with an equivalent minimization problem in which the optimal solution of this problem is the approximate solution of the original FDE. Moreover, since the error of this approach is minimized, the approximate solutions are the best solutions for the original problem. We employ this approximation to get numerical solution of a system of FDEs which has been used for modelling HIV infection of CD4 ${ }^{+} \mathrm{T}$ cells.

The discussion of paper will be as follows: in the next section, we express the fractional HIV model and introduce the notations that used in the rest of this paper. In Section 3, we design an efficient approach to approximate the fractional derivative and use it in our numerical method for solving FDEs. Some numerical examples are displayed in Section 4. Finally, conclusions are included in the last section.

\section{The Problem}

Consider the following fractional-order differential equation model of HIV infection of CD4 ${ }^{+} \mathrm{T}$ cells [25]:

$$
\left\{\begin{array}{l}
D^{\alpha} T=s-\mu_{T} T+r T\left(1-\frac{T+I}{T_{\max }}\right)-k_{V} T, \\
D^{\alpha} I=k_{I}^{\prime} V T-\mu_{I} I, \\
D^{\alpha} V=N \mu_{b} I-k_{I} V T-\mu_{V} V,
\end{array}\right.
$$

with the initial conditions $T(0)=T_{0}, I(0)=0$ and $V(0)=0$, in which the parameter values reported by Table 1.

Following Theorem 1 of [25], we note that (1) along with its initial conditions possesses a unique solution which is non-negative. Throughout this paper, we set $D^{\alpha} \quad(0<\alpha<1)$ as the Riemann-Liouville derivative of order $\alpha$ defined by [26]:

$$
D^{\alpha} f(t)=\frac{1}{\Gamma(1-\alpha)} \frac{\mathrm{d}}{\mathrm{d} t} \int_{0}^{t}(t-\tau)^{-\alpha} f(\tau) \mathrm{d} \tau
$$

The aim of this paper is to extend the application of the AVK approach to solve a fractional order model for this HIV infection model of $\mathrm{CD}^{+} \mathrm{T}$ cells. So, in the next section, at first we convert the original FDE to an

\begin{tabular}{|c|c|}
\hline Parameter & Value/unit \\
\hline$\mu_{T} \quad\left(\right.$ Natural death rate of $\left.\mathrm{CD}^{+} \mathrm{T}\right)$ & 0.02 day $^{-1}$ \\
\hline$\mu_{I} \quad$ (Blanket death rate of infected $\left.\mathrm{CD} 4^{+} \mathrm{T}\right)$ & 0.26 day $^{-1}$ \\
\hline$\mu_{v} \quad$ (Death rate of free virus) & 2.4 day $^{-1}$ \\
\hline$\mu_{b} \quad$ (Lytic death rate for infected cells) & 0.24 day $^{-1}$ \\
\hline$k_{I} \quad$ (Rate $\mathrm{CD}^{+} \mathrm{T}$ become infected with virus) & $2.4 \times 10^{-5} \mathrm{~mm}^{-3} \cdot$ day $^{-1}$ \\
\hline$k_{I}^{\prime} \quad$ (Rate infected cells become active) & $2 \times 10^{-5} \mathrm{~mm}^{-3} \cdot \mathrm{day}^{-1}$ \\
\hline$r$ (Rowth rate of $\mathrm{CD} 4^{+} \mathrm{T}$ population) & 0.03 day $^{-1}$ \\
\hline$N$ (Number of virions produced by infected CD $4^{+} \mathrm{T}$ ) & Varies \\
\hline$T_{\max }$ (Maximal population level of $\mathrm{CD}^{+} \mathrm{T}$ ) & $1500 \mathrm{~mm}^{-3}$ \\
\hline$s$ (Source term for uninfected $\mathrm{CD} 4^{+} \mathrm{T}$ ) & $10 \mathrm{day}^{-1} \cdot \mathrm{mm}^{-3}$ \\
\hline$T_{0}$ (CD4 ${ }^{+} \mathrm{T}$ population for HIV-negative persons) & $1000 \mathrm{~mm}^{-3}$ \\
\hline
\end{tabular}

Table 1. Variables and parameters for HIV infection model. 
optimization problem based on minimization of error. By discretizing the new problem and approximating the Riemann-Liouville fractional derivative by a finite difference method, we obtaine the best approximate solution of the original FDE.

\section{AVK Approach for Solving Approximately FDEs}

Consider a general system of FDEs as follows:

$$
\begin{aligned}
& D^{\alpha} x(t)=g(x, t), \\
& x(0)=x_{0},
\end{aligned}
$$

where $D^{\alpha} \quad(0<\alpha<1)$ is the Riemann-Liouville derivative of order $\alpha, g$ is an riemann integrable time varying function, $g: A \times[0,1] \rightarrow \mathbb{R}^{n}, t \in[0,1] \subseteq \mathbb{R}$ and $A$ is a compact subset in $\mathbb{R}^{n}$. Also

$x(t)=\left(x_{1}(t), \cdots, x_{n}(t)\right) \in A$ called the state variable. We want to obtain an approximate solution of problem (3). Therefore, we need the following definition.

Definition 1. For problem (3) we define the following functional $E($.$) that is called the total error$ functional:

$$
E\left(D^{\alpha} x, x, t\right)=\int_{0}^{1}\left\|D^{\alpha} x(t)-g(x, t)\right\| \mathrm{d} t,
$$

where $E: A \times[0,1] \rightarrow \mathbb{R}$ is a non-negative functional, $\|\cdot\|$ is any norm in $\mathbb{R}^{n}$ space, such as $\|\cdot\|_{1}$ where is defined as follows:

$$
\|x(t)\|_{1}=\left\|\left(x_{1}(t), \cdots, x_{n}(t)\right)\right\|_{1}=\sum_{i=1}^{n}\left|x_{i}(t)\right| .
$$

Here, we convert the problem (4) to a nonlinear programming (NLP) as follow:

$$
\left\{\begin{array}{lc}
\min & E\left(D^{\alpha} x, x, t\right) \\
\text { s.t } & x(0)=x_{0}, \\
& (x, t) \in A \times[0,1] .
\end{array}\right.
$$

Now, to reach the approximating solution for the original problem (3) it is sufficient to solve the minimization problem (6). Hence, we need the following mean theorem [27] and corollary.

Theorem 1. Let $h$ be a nonnegative continuous function on $[a, b]$, the necessary and sufficient condition for $\int_{a}^{b} h \mathrm{~d} t=0$ is that $h \equiv 0$, on $[a, b]$.

${ }^{a}$ Corollary 1. Necessary and sufficient condition for the trajectory $x(t)$ to be a solution of system (3) is that the optimal solution of (6) has zero objective function.

To develop the numerical solution of problem (6) approximately, we defined the grid size in time by $\delta t=\frac{1}{m}$ for some positive integer $m$, so the grid points in the time interval $[0,1]$ is given by $t_{k}=k \delta t, k=1, \cdots, m$. In order to illustrate the numerical approach better, we introduce the following notations:

$$
x_{i}^{k}=x_{i}\left(t_{k}\right), g_{i}^{k}=g\left(x_{i}^{k}, t_{k}\right), i=1, \cdots, n, k=1, \cdots, m .
$$

By the above notations, problem (6) is now approximated by the following optimization problem:

$$
\left\{\begin{array}{l}
\min J(x, t)=\sum_{i=1}^{n} \sum_{k=1}^{m} \int_{t_{k-1}}^{t_{k}}\left|D^{\alpha} x_{i}(t)-g\left(t, x_{i}(t)\right)\right| \mathrm{d} t \\
\text { s.t } \quad x(0)=x_{0}, x_{i}^{k} \in A \subseteq \mathbb{R}^{n}, t \in\left(t_{k-1}, t_{k}\right) \subseteq \mathbb{R} .
\end{array}\right.
$$

By using the ending point in any subinterval for approximating integrals, problem (7) is now approximated by the following optimization problem:

$$
\min _{x} J\left(x_{i}, t\right)=\sum_{i=1}^{n} \sum_{k=1}^{m} \frac{1}{m}\left|D^{\alpha} x_{i}^{k}-g_{i}^{k}\right|
$$


Now, we approximate fractional derivative $D^{\alpha} x_{i}$ as follows:

$$
D^{\alpha} x_{i}=\frac{1}{\Gamma(1-\alpha)} \frac{\mathrm{d}}{\mathrm{d} t} \int_{0}^{t} \frac{x_{i}(\tau)}{(t-\tau)^{\alpha}} \mathrm{d} \tau .
$$

Define $y(t)=\int_{0}^{t} x_{i}(\tau)(t-\tau)^{-\alpha} \mathrm{d} \tau$. Then, Equation (9) yields to

$$
D^{\alpha} x_{i}=\frac{1}{\Gamma(1-\alpha)} \frac{\mathrm{d}}{\mathrm{d} t} y(t) .
$$

In order to better illustrate the numerical approach, we also introduce the following difference operator:

$$
\frac{\mathrm{d}}{\mathrm{d} t} y(t) \cong \frac{y(t+\delta t)-y(t)}{\delta t}=m(y(t+\delta t)-y(t)) .
$$

Then,

$$
\frac{\mathrm{d}}{\mathrm{d} t} y(t)=m\left(\int_{0}^{t+\delta t} x_{i}(\tau)(t+\delta t-\tau)^{-\alpha} \mathrm{d} \tau-\int_{0}^{t} x_{i}(\tau)(t-\tau)^{-\alpha} \mathrm{d} \tau\right) .
$$

Hence $\delta t$ or sampling time is very important, and must be chosen small, so the number of partitions is great. This is a trade off between sampling time and speed of problem solving. Using again trapezoidal rule in any subinterval for approximating integrals, except for the last interval that we use the midpoint approximation, and suppose $t_{h}=\frac{h}{m}, x_{i}^{h}=x_{i}\left(\frac{h}{m}\right)$ for $h=1,2, \cdots, k$. Therefore,

$$
\begin{aligned}
\frac{\mathrm{d}}{\mathrm{d} t} y\left(t_{k}\right)= & m\left(\int_{0}^{t_{k+1}} x_{i}(\tau)\left(t_{k+1}-\tau\right)^{-\alpha} \mathrm{d} \tau-\int_{0}^{t_{k}} x_{i}(\tau)\left(t_{k}-\tau\right)^{-\alpha} \mathrm{d} \tau\right) \\
\cong & m\left(\sum_{h=1}^{k+1} \frac{\int_{h-1}^{\frac{h}{m}} x_{i}}{m}(\tau)\left(t_{k+1}-\tau\right)^{-\alpha} \mathrm{d} \tau-\sum_{h=1}^{k} \int_{\frac{h-1}{m}}^{\frac{h}{m}} x_{i}(\tau)\left(t_{k}-\tau\right)^{-\alpha} \mathrm{d} \tau\right) \\
\cong & m\left\{\left(\sum_{h=1}^{k} \frac{1}{m} x_{i}^{h}\left(t_{k+1}-t_{h}\right)^{-\alpha}+\frac{1}{m} x_{i}\left(\frac{2 k+1}{2 m}\right)\left(t_{k+1}-\frac{2 k+1}{2 m}\right)^{-\alpha}\right)\right. \\
& \left.-\left(\sum_{h=1}^{k-1} \frac{1}{m} x_{i}^{h}\left(t_{k}-t_{h}\right)^{-\alpha}+\frac{1}{m} x_{i}\left(\frac{2 k-1}{2 m}\right)\left(t_{k}-\frac{2 k-1}{2 m}\right)^{-\alpha}\right)\right\} .
\end{aligned}
$$

Thus, we simply get problem (8) in the following form:

$$
\min _{x_{i}} \sum_{i=1}^{n} \sum_{k=1}^{m} \frac{1}{m}\left|\frac{1}{\Gamma(1-\alpha)}\left\{\sum_{h=1}^{k} x_{i}^{h}\left(t_{k+1}-t_{h}\right)^{-\alpha}-\sum_{h=1}^{k-1} x_{i}^{h}\left(t_{k}-t_{h}\right)^{-\alpha}+\left(\frac{1}{2 m}\right)^{-\alpha}\left(x_{i}^{k+\frac{1}{2}}-x_{i}^{k-\frac{1}{2}}\right)\right\}-g_{i}^{k}\right|,
$$

in which, $x_{i}^{k \pm \frac{1}{2}}=x_{i}\left(t_{k} \pm \frac{\delta t}{2}\right)$ for $k=1,2, \cdots, m$.

We solved this optimization problem by linear programming (LP) formulation which is done in what follows.

Lemma 1. Let pairs $\left(v_{i}^{*}, u_{i}^{*}\right), \quad i=1,2, \cdots, m$, be the optimal solutions of the following LP problem:

$$
\left\{\begin{array}{l}
\min \sum_{i=1}^{m} v_{i} \\
\text { s.t } v_{i} \geq u_{i}, v_{i} \geq-u_{i}, v_{i} \geq 0, u_{i} \in I .
\end{array}\right.
$$

where $I$ is a compact set. Then $u_{i}^{*}, \quad i=1,2, \cdots, m$, is the optimal solution of the following NLP problem:

$$
\min _{u \in I} \sum_{i=1}^{m}\left|u_{i}\right| \text {. }
$$

Proof. Since, $\left(v_{i}^{*}, u_{i}^{*}\right), i=1,2, \cdots, m$, is the optimal solution of the LP problem, so they satisfy the con- 
straints. Thus there is $v_{i}^{*} \geq u_{i}^{*}$ and $v_{i}^{*} \geq-u_{i}^{*}$ for $i=1,2, \cdots, m$. Hence, $\left|u_{i}^{*}\right| \leq v_{i}^{*}, \quad i=1,2, \cdots, m$, and so $\sum_{i=1}^{m}\left|u_{i}^{*}\right| \leq \sum_{i=1}^{m} v_{i}^{*}$. Now, let there exist $\bar{u}_{i}^{*} \in I, \quad i=1,2, \cdots, m$, such that $\sum_{i=1}^{m}\left|\bar{u}_{i}^{*}\right|<\sum_{i=1}^{m}\left|u_{i}^{*}\right|$. Define, $\bar{v}_{i}^{*}=\left|\bar{u}_{i}^{*}\right|$ for $i=1,2, \cdots, m$. Then $\bar{v}_{i}^{*} \geq \bar{u}_{i}^{*}$ and $\bar{v}_{i}^{*} \geq-\bar{u}_{i}^{*}$. Moreover, $\sum_{i=1}^{m} \bar{v}_{i}^{*}=\sum_{i=1}^{m}\left|\bar{u}_{i}^{*}\right|$ and hence

$$
\sum_{i=1}^{m} \bar{v}_{i}^{*}=\sum_{i=1}^{m}\left|\bar{u}_{i}^{*}\right|<\sum_{i=1}^{m}\left|u_{i}^{*}\right|<\sum_{i=1}^{m} v_{i}^{*}
$$

So $\sum_{i=1}^{m} \bar{v}_{i}^{*}<\sum_{i=1}^{m} v_{i}^{*}$, which is a contradiction. See [28] more details.

Now, by lemma 1, problem (14) can be converted to the following equivalent LP problem:

$$
\begin{aligned}
& \min \sum_{i=1}^{n} \sum_{k=1}^{m} \frac{1}{m \Gamma(1-\alpha)} \mu_{i}^{k} \\
& \text { s.t }-\mu_{i}^{k}+\sum_{h=1}^{k} x_{i}^{h}\left(t_{k+1}-t_{h}\right)^{-\alpha}-\sum_{h=1}^{k-1} x_{i}^{h}\left(t_{k}-t_{h}\right)^{-\alpha}+\left(\frac{1}{2 m}\right)^{-\alpha}\left(x_{i}^{k+\frac{1}{2}}-x_{i}^{k-\frac{1}{2}}\right) \leq \Gamma(1-\alpha) g_{i}^{k} \\
& -\mu_{i}^{k}-\sum_{h=1}^{k} x_{i}^{h}\left(t_{k+1}-t_{h}\right)^{-\alpha}+\sum_{h=1}^{k-1} x_{i}^{h}\left(t_{k}-t_{h}\right)^{-\alpha}-\left(\frac{1}{2 m}\right)^{-\alpha}\left(x_{i}^{k+\frac{1}{2}}-x_{i}^{k-\frac{1}{2}}\right) \leq-\Gamma(1-\alpha) g_{i}^{k} \\
& \quad i=1,2, \cdots, n, k=1,2, \cdots, m .
\end{aligned}
$$

By obtaining the solution of this problem, we recognize the value of unknown admissible $x_{i}^{k}, i=1,2, \cdots, n$ and $k=1,2, \cdots, m$.

\section{Numerical Examples}

In this section, we give some numerical examples and apply the method presented in the last sections for solving them. Moreover, we extend this approach for approximately solving a model of HIV infection of $\mathrm{CD} 4^{+} \mathrm{T}$ cells with therapy effect including a system of FDEs. These test problems demonstrate the validity and efficiency of this approximation.

Example 1. As first example, we compute $D^{\alpha} x(t)$, with $\alpha=\frac{1}{2}$, for $x(t)=t^{4}$. The exact formulas of the derivatives are derived from

$$
D^{0.5}\left(t^{s}\right)=\frac{\Gamma(s+1)}{\Gamma(s+1-0.5)} t^{s-0.5}
$$

Figure 1 shows the results by using approximation (10)-(13) for $\alpha=0.5$ and various choices of $m$.

Now, assume that $\bar{x}_{i}\left(t_{k}\right), i=1, \cdots, n, k=1, \cdots, m$ and $x_{i}\left(t_{k}\right)$ are the approximated and exact solutions of system (3), respectively. We defined the absolute error of approximation as follow:

$$
E=\max _{k}\left(\left\|x_{i}\left(t_{k}\right)-\bar{x}_{i}\left(t_{k}\right)\right\|_{1}\right), i=1, \cdots, n, k=1,2, \cdots, m .
$$

In this example, the maximum absolute errors computed by Equation (16) for $\alpha=0.5$ and various choices of $m$, has been shown in Table 2 .

Example 2. Consider the following initial value problem:

$$
D^{0.5} x(t)=t^{2}-x(t)+\frac{2}{\Gamma(2.5)} t^{\frac{3}{2}}
$$

with initial condition $x(0)=0$.

We know that $D^{0.5}\left(t^{2}\right)=\frac{2}{\Gamma(2.5)} t^{\frac{3}{2}}$. Therefore, the analytic solution for system (17) is $x(t)=t^{2}$. Now we expand the fractional derivative up to the problem (15). The solution is drawn in Figures 2-4 for $m=20,50,100$ and $\alpha=0.1,0.5,0.99$. 


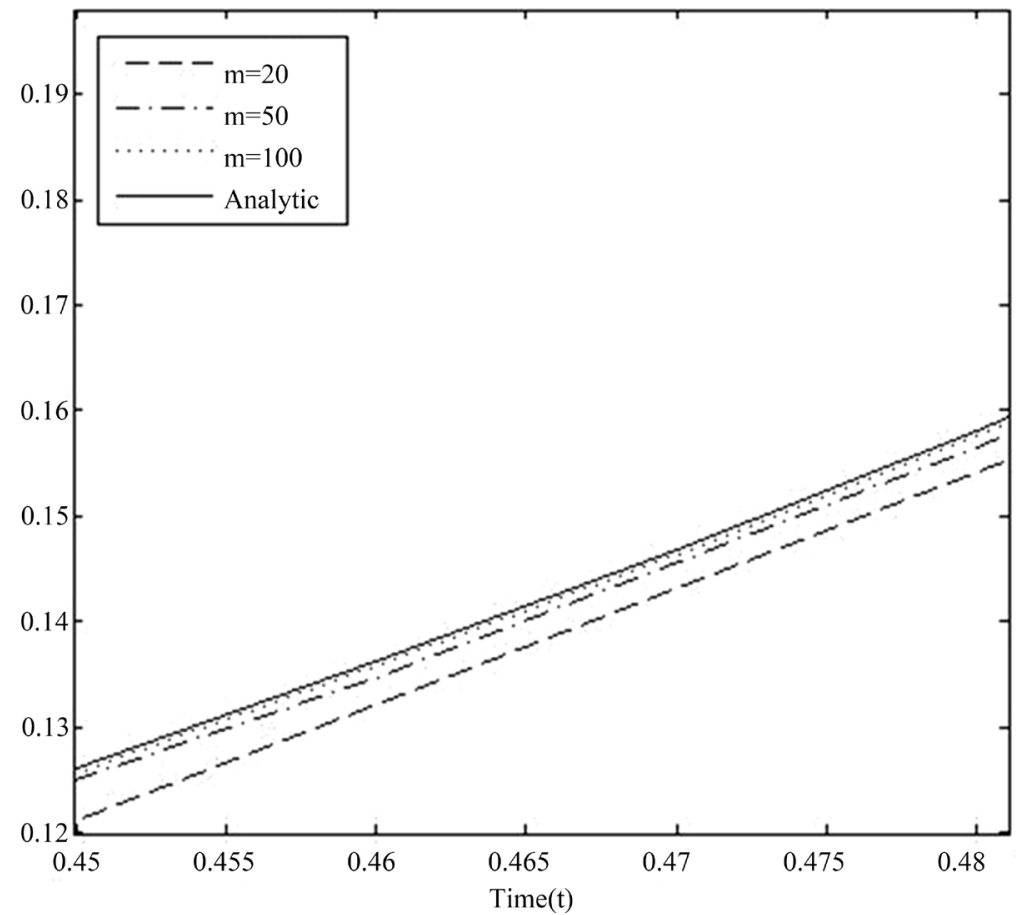

Figure 1. Analytic solution and numerical approximation (10), with various choices of $m$ and $\alpha=0.5$, for Example 1 .

Table 2. Maximum absolute error for Example 1.

\begin{tabular}{cc}
\hline & $E_{\text {appro }}$ \\
\hline 5 & $2.65757 \times 10^{-3}$ \\
10 & $6.47115 \times 10^{-4}$ \\
20 & $4.09595 \times 10^{-4}$ \\
40 & $9.77932 \times 10^{-5}$ \\
50 & $2.27352 \times 10^{-5}$ \\
100 & $5.04375 \times 10^{-6}$ \\
\hline
\end{tabular}

In the case of $\alpha=0.1,0.5,0.99$, the maximum absolute errors (16) with various choices of $m$ is shown in Table 3.

From numerical results we can indicate that the solution of FDE approaches to the solution of integer order differential equation, whenever $\alpha$ approaches to its integer value.

Example 3. Consider the following FDE:

$$
{ }_{0} D_{t}^{\alpha} x(t)=\frac{2}{\Gamma(3-\alpha)} t^{2-\alpha}-\frac{1}{\Gamma(2-\alpha)} t^{1-\alpha}-x(t)+t^{2}-t,
$$

where $0<\alpha \leq 1$ and $x(0)=0$.

The exact solution of this equation is $x(t)=t^{2}-t$. In Figure 5 \& Figure 6, we compare the exact solution with the numerical approximation (15) for two values of $m$ and $\alpha=0.5$.

Table 4 shows the exact solution and the approximate solution for equation (18) by solving problem (15) for $m=100$ and $\alpha=0.5,0.99$. The results compare well with those obtained in [29].

Example 4. Now we want to solve the fractional-order differential equation model of HIV infection of $\mathrm{CD} 4{ }^{+} \mathrm{T}$ cells (1) For the parameter values given in Table 1. The system (1) can be expressed in a vector form as follows: 


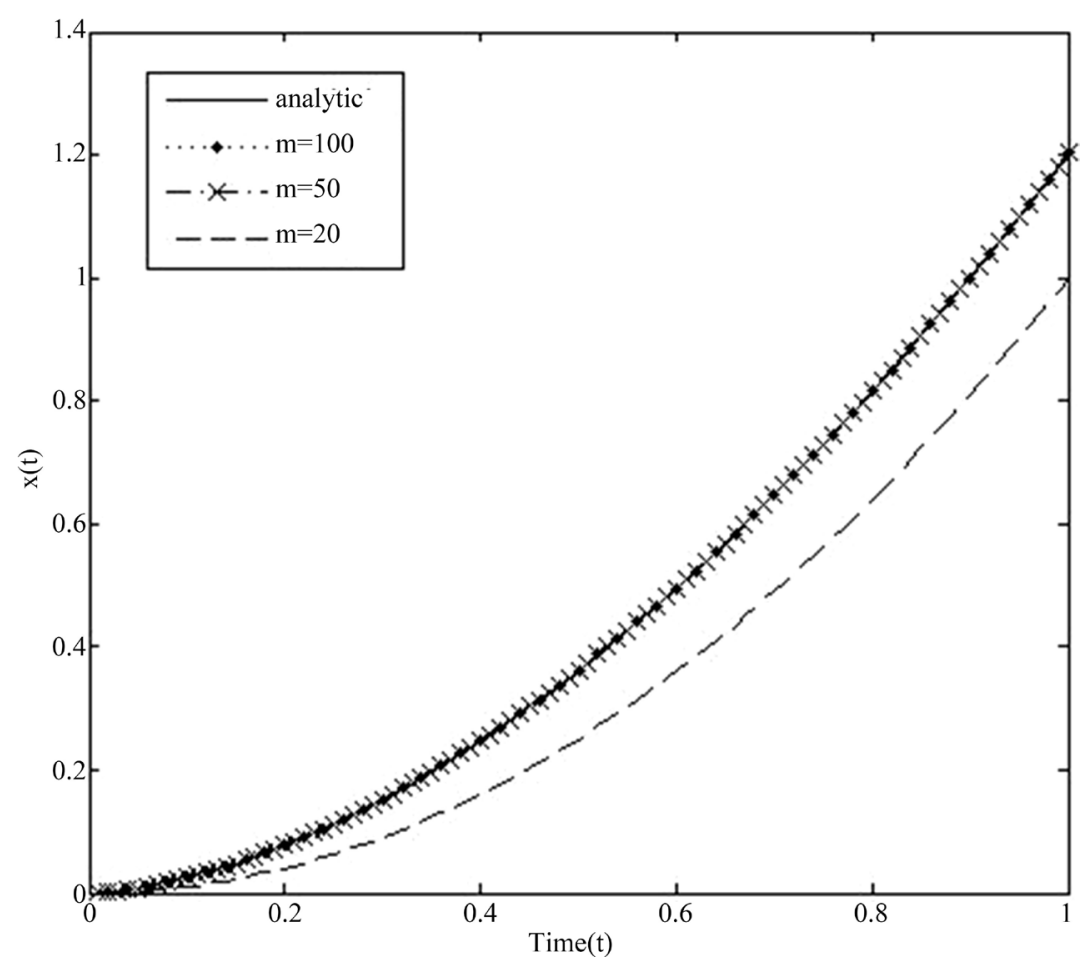

Figure 2. Exact and approximation solutions for problem in Example 2 with $\alpha=0.1$ and different values of $m$.

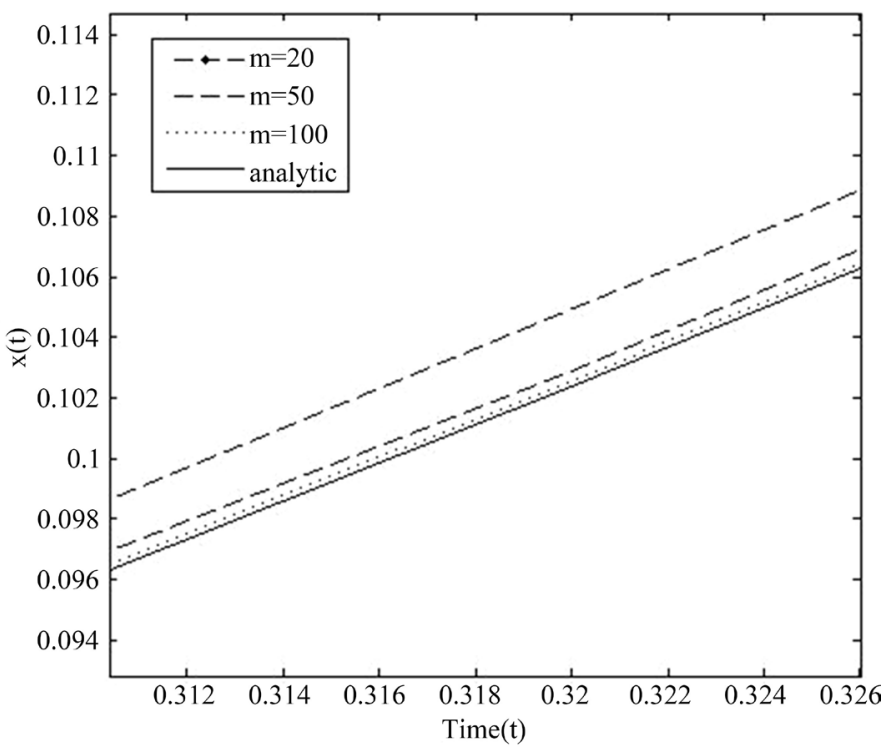

Figure 3. Exact and approximation solutions for problem in Example 2 with $\alpha=0.5$ and different values of $m$.

$$
D^{\alpha} x(t)=g(t, x(t)),
$$

where $x(t)=(T(t), I(t), V(t))$ is the state vector and

$$
x(0)=\left(T_{0}, 0,0\right) .
$$

For numerical simulations we assumed 350 days for treatment period. With the change of variables $t=350 \tau$, 


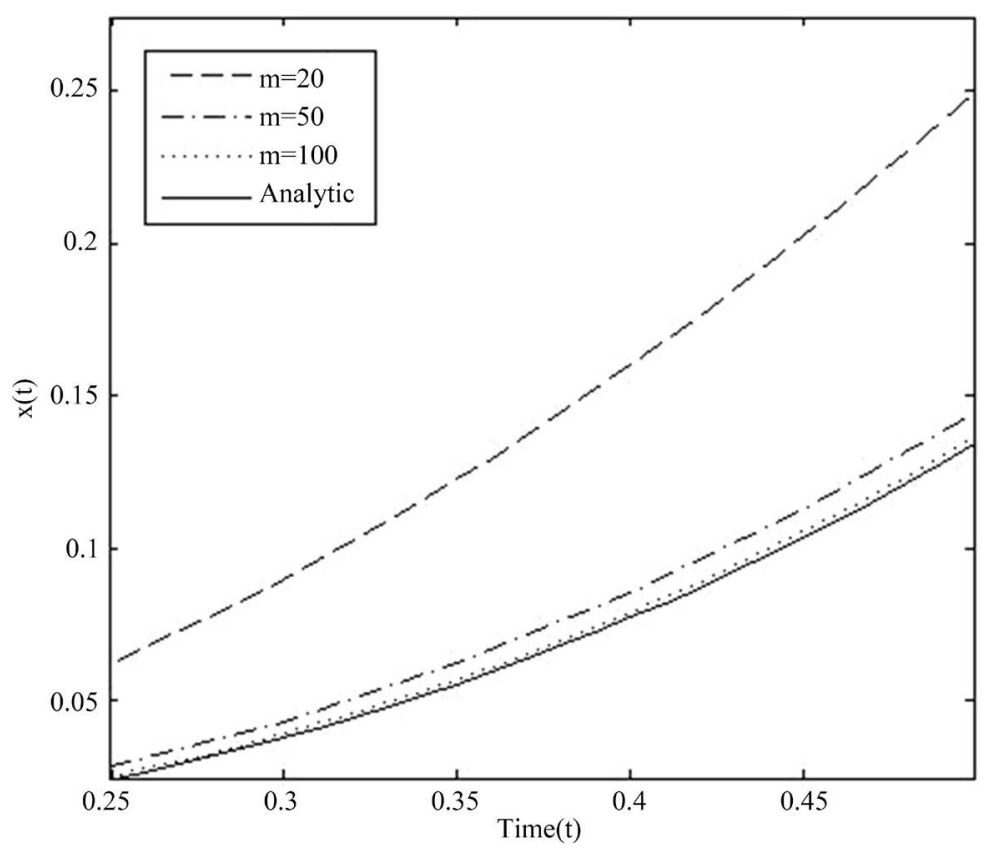

Figure 4. Exact and approximation solutions for problem in Example 2 with $\alpha=0.99$ and different values of $m$.

Table 3. Maximum absolute error for different values of $\alpha, m$ for Example 2.

\begin{tabular}{cccc}
\hline$m$ & $\alpha=0.1$ & $\alpha=0.5$ & $\alpha=0.99$ \\
\hline 10 & 0.26227 & $2.61278 \times 10^{-4}$ & $2.65757 \times 10^{-3}$ \\
20 & 0.11574 & $2.14695 \times 10^{-4}$ & $6.47115 \times 10^{-4}$ \\
40 & 0.08285 & $1.99832 \times 10^{-4}$ & $4.09595 \times 10^{-4}$ \\
50 & 0.02621 & $1.55710 \times 10^{-4}$ & $9.77932 \times 10^{-5}$ \\
100 & 0.00748 & $1.16467 \times 10^{-4}$ & $2.27352 \times 10^{-5}$ \\
\hline
\end{tabular}

Table 4. Numerical values with $\alpha=0.5,0.99$ and $m=100$ for Example 3 .

\begin{tabular}{ccccc}
\hline$t$ & $X_{\text {appro }}(\alpha=0.5)$ & $X_{\text {exact }}$ & $X_{\text {appro }}(\alpha=0.99)$ & $X_{\text {exact }}$ \\
\hline 0.0 & 0.000000 & 0.000000 & 0.000000 & 0.000000 \\
0.1 & -0.089978 & -0.090000 & -0.089586 & -0.090000 \\
0.2 & -0.159889 & -0.160000 & -0.159688 & -0.160000 \\
0.3 & -0.209891 & -0.210000 & -0.209707 & -0.2100000 \\
0.4 & -0.239974 & -0.240000 & -0.239787 & -0.240000 \\
0.5 & -0.249896 & -0.250000 & -0.249738 & -0.2500000 \\
0.6 & -0.239998 & -0.240000 & -0.239795 & -0.240000 \\
0.7 & -0.199879 & -0.210000 & -0.209830 & -0.210000 \\
0.8 & -0.160109 & -0.160000 & -0.159897 & -0.160000 \\
0.9 & -0.096390 & -0.090000 & -0.100098 & -0.090000
\end{tabular}




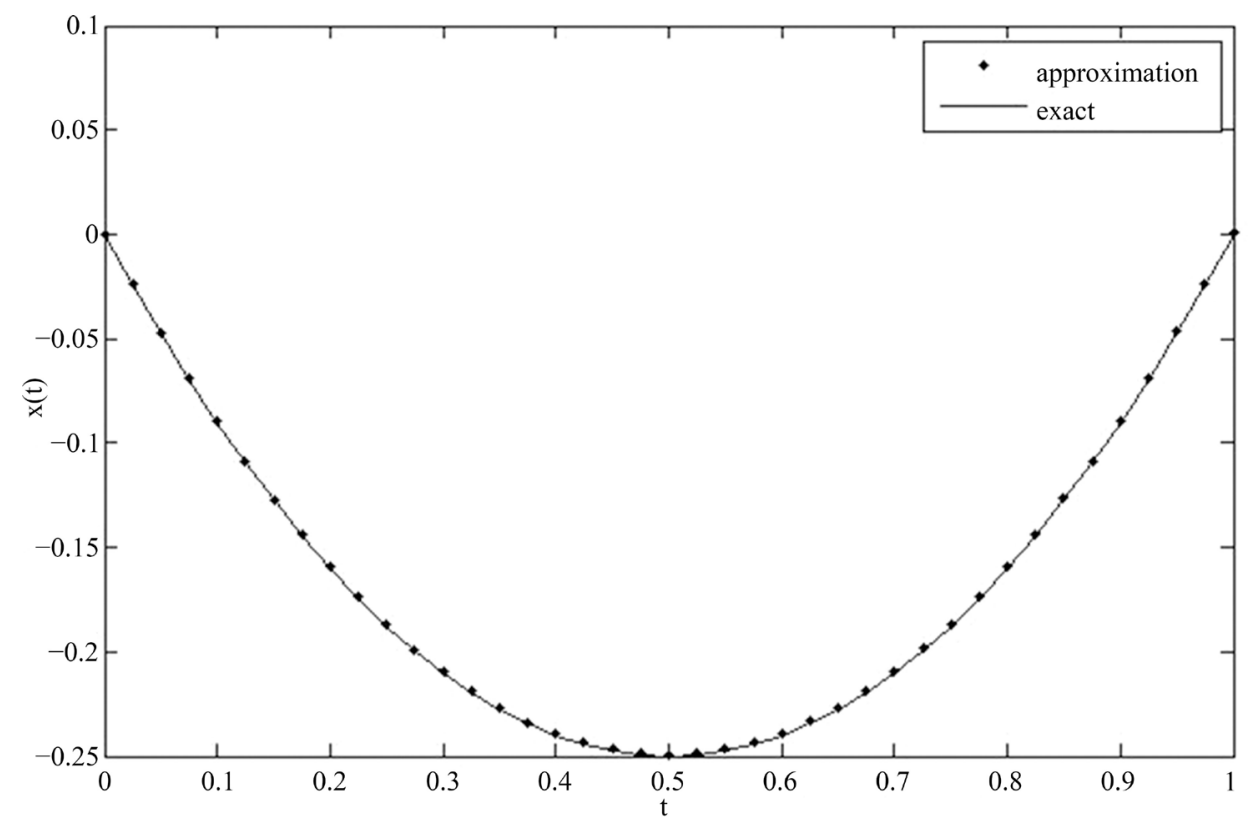

Figure 5. Analytic solution and numerical approximation (15) for Example 3 for $m=40$.

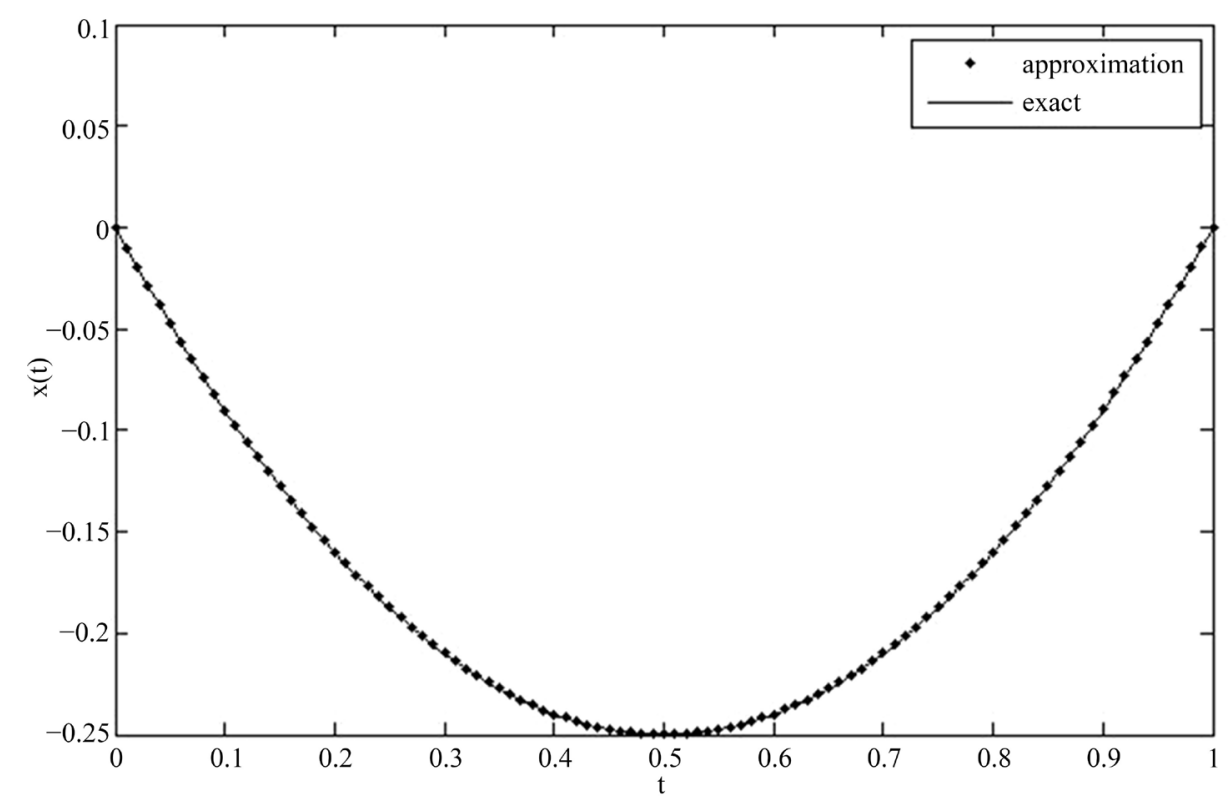

Figure 6. Analytic solution and numerical approximation (15) for Example 3 for $m=100$.

we converted period $t \in[0,350]$ to $\tau \in[0,1]$. Based on concepts was said in the previous section, the key to the derivation of the approach is to replace the system (19) by the following equivalent optimization problem:

$$
\begin{aligned}
& \min \sum_{k=1}^{m} \int_{\tau_{i-1}}^{\tau_{i}}\left\{\left|D^{\alpha} T(350 \tau)-s-\mu_{T} T(350 \tau)+r T(350 \tau)\left(1-\frac{T(350 \tau)+I(350 \tau)}{T_{\max }}\right)-k_{V} T(350 \tau)\right|\right. \\
& +\left|D^{\alpha} I(350 \tau)-k_{1}^{\prime} V(350 \tau) T(350 \tau)-\mu_{I} I(350 \tau)\right| \\
& \left.+\left|D^{\alpha} V(350 \tau)-N \mu_{b} I(350 \tau)-k_{I} V(350 \tau) T(350 \tau)-\mu_{V} V(350 \tau)\right|\right\} \mathrm{d} \tau,
\end{aligned}
$$


Table 5. Maximum absolute error for $m=100$ and different values of $\alpha$ for Example 4 .

\begin{tabular}{cccccc}
\hline$P$ & $\alpha=0.5$ & $\alpha=0.6$ & $\alpha=0.7$ & $\alpha=0.8$ & $\alpha=0.9$ \\
$T$ & $8.0224 E-5$ & $1.0253 E-6$ & $2.2102 E-7$ & $2.8532 E-8$ & $1.0929 E-9$ \\
$I$ & $1.1031 E-5$ & $1.9756 E-6$ & $1.1102 E-8$ & $2.4527 E-8$ & $1.7608 E-9$ \\
$V$ & $1.2226 E-5$ & $2.7756 E-7$ & $1.19553 E-7$ & $2.6392 E-9$ & $1.2079 E-9$ \\
\hline
\end{tabular}

with the initial condition (20). To solve this optimization problem, by approximating integrals as before, we transformed (21) to a discretized problem in the following form:

$$
\begin{aligned}
& \min \frac{1}{m} \sum_{k=1}^{m}\left\{\left|D^{\alpha} T\left(350 \tau_{k}\right)-s-\mu_{T} T\left(350 \tau_{k}\right)+r T\left(350 \tau_{k}\right)\left(1-\frac{T\left(350 \tau_{k}\right)+I\left(350 \tau_{k}\right)}{T_{\max }}\right)-k_{V} T\left(350 \tau_{k}\right)\right|\right. \\
& +\left|D^{\alpha} I\left(350 \tau_{k}\right)-k_{1}^{\prime} V\left(350 \tau_{k}\right) T\left(350 \tau_{k}\right)-\mu_{I} I\left(350 \tau_{k}\right)\right| \\
& \left.+\left|D^{\alpha} V\left(350 \tau_{k}\right)-N \mu_{b} I\left(350 \tau_{k}\right)-k_{I} V\left(350 \tau_{k}\right) T\left(350 \tau_{k}\right)-\mu_{V} V\left(350 \tau_{k}\right)\right|\right\} .
\end{aligned}
$$

In problem (21) and (22), the factor 350 is omitted because of having no effect on the solution of it. Then, the minimum problem (22) converted to a linear programming problem with the following change of variables:

$$
\begin{aligned}
& \min \frac{1}{m} \sum_{k=1}^{m} u_{k}+v_{k}+r_{k}+e_{k}+w_{k}+q_{k} \\
& \text { s.t. } D^{\alpha} T\left(350 \tau_{k}\right)-s-\mu_{T} T\left(350 \tau_{k}\right)+r T\left(350 \tau_{k}\right)\left(1-\frac{T\left(350 \tau_{k}\right)+I\left(350 \tau_{k}\right)}{T_{\max }}\right)-k_{V} T\left(350 \tau_{k}\right)=u_{k}-v_{k}, \\
& D^{\alpha} I\left(350 \tau_{k}\right)-k_{1}^{\prime} V\left(350 \tau_{k}\right) T\left(350 \tau_{k}\right)-\mu_{I} I\left(350 \tau_{k}\right)=r_{k}-e_{k}, \\
& D^{\alpha} V\left(350 \tau_{k}\right)-N \mu_{b} I\left(350 \tau_{k}\right)-k_{I} V\left(350 \tau_{k}\right) T\left(350 \tau_{k}\right)-\mu_{V} V\left(350 \tau_{k}\right)=w_{k}-q_{k}, \\
& T(0)=T_{0}, I(0)=V(0)=0, u_{k}, v_{k}, r_{k}, e_{k}, w_{k}, q_{k} \geq 0 .
\end{aligned}
$$

Now, we approximate fractional derivatives from (10)-(13). Our approach introduces an approximate solution for the fractional HIV model based on minimization the total error. The maximum absolute errors (16) with $m=$ 100 and different values of $\alpha$ that shown in Table 5, confirmed the efficacy of our approach in comparison with the result obtained by [25].

\section{Conclusions}

In this paper, the finite difference method discrete time AVK approach has been successfully used for finding the solutions of a system of FDEs such as a model for HIV infection of $\mathrm{CD} 4^{+} \mathrm{T}$ cells. Our approach introduces an approximate solution for the FDEs based on the minimization of the total error. In the suggested method, the original problem reduces to an optimization problem. By discretizing the new problem and solving it, we obtain the best approximate solution of the original problem. Results represent a unifying approach for numerical approximation of differential equations of fractional order. Since this method is not based on point to point error, but according to its results, it is clear that there is no difference between the exact and approximate solutions in point to point case.

Three numerical examples are given and the results are compared with the exact solutions and with the other methods. It is shown that, as the order of fractional derivatives approaches to 1 , the numerical solutions for the FDEs approach the clasicall solutions of the problem. Then we use this technique for finding approximate solutions of FDEs system of a model for HIV infection of $\mathrm{CD} 4^{+} \mathrm{T}$ cells. The result demonstrates the validity of the approach.

\section{References}

[1] Barkai, E., Metzler, R. and Klafter, J. (2000) From Continuous Time Random Walks to the Fractional Fokker-Planck 
Equation. Physical Review E, 61, 132. http://dx.doi.org/10.1103/PhysRevE.61.132

[2] Bhrawy, A.H., Doha, E.H., Tenreiro Machado, J.A. and Ezz-Eldien, S.S. (2015) An Efficient Numerical Scheme for Solving Multi-Dimensional Fractional Optimal Control Problems with a Quadratic Performance Index. Asian Journal of Control, 17, 2389-2402. http://dx.doi.org/10.1002/asjc.1109

[3] Podlubny, I. (1998) Fractional Differential Equations: An Introduction to Fractional Derivatives, Fractional Differential Equations, to Methods of Their Solution and Some of Their Applications. Vol. 198, Academic Press, Mathematics in Science and Engineering, 366.

[4] Magin, R.L. (2006) Fractional Calculus in Bioengineering. Vol. 149, Begell House Publishers, Redding.

[5] Raberto, M., Scalas, E. and Mainardi, F. (2002) Waiting-Times and Returns in High-Frequency Financial Data: An Empirical Study. Physica A: Statistical Mechanics and its Applications, 314, 749-755.

[6] Tricaud, C. and Chen, Y.Q. (2010) An Approximate Method for Numerically Solving Fractional Order Optimal Control Problems of General Form. Computers \& Mathematics with Applications, 59, 1644-1655. http://dx.doi.org/10.1016/j.camwa.2009.08.006

[7] Zamani, M., Karimi, G. and Sadati, N. (2007) Fopid Controller Design for Robust Performance Using Particle Swarm Optimization. Fractional Calculus and Applied Analysis, 10, 169-188.

[8] Bagley, R.L. and Torvik, P.J. (1983) A Theoretical Basis for the Application of Fractional Calculus to Viscoelasticity. Journal of Rheology, 27, 201-210. http://dx.doi.org/10.1122/1.549724

[9] Anastasio, T.J. (1994) The Fractional-Order Dynamics of Bainstem Vestibulo-Oculomotor Neurons. Biological Cybernetics, 72, 69-79. http://dx.doi.org/10.1007/BF00206239

[10] Liu, F., Anh, V. and Turner, I. (2004) Numerical Solution of the Space Fractional Fokker-Planck Equation. Journal of Computational and Applied Mathematics, 166, 209-219. http://dx.doi.org/10.1016/j.cam.2003.09.028

[11] Shen, S., Liu, F., Anh, V. and Turner, I. (2008) The Fundamental Solution and Numerical Solution of the Riesz Fractional Advection-Dispersion Equation. IMA Journal of Applied Mathematics, 73, 850-872. http://dx.doi.org/10.1093/imamat/hxn033

[12] Bhrawy, A.H., Baleanu, D. and Assas, L.M. (2013) Efficient Generalized Laguerre-Spectral Methods for Solving Multi-Term Fractional Differential Equations on the Half Line. Journal of Vibration and Control, 20, 973-985.

[13] Pooseh, S., Almeida, R. and Torres, D. (2013) Numerical Approximations of Fractional Derivatives with Applications. Asian Journal of Control, 15.3, 698-712. http://dx.doi.org/10.1002/asjc.617

[14] Grahovac, N.M. and Spasic, D.T. (2013) Multivalued Fractional Differential Equations as a Model for an Impact of Two Bodies. Journal of Vibration and Control, 20, 1017-1032.

[15] Saadatmandi, A. and Dehghan, M. (2011) A Legendre Collocation Method for Fractional Integro-Differential Equations. Journal of Vibration and Control, 17, 2050-2058. http://dx.doi.org/10.1177/1077546310395977

[16] Kayedi-Bardeh, A., Eslahchi, M.R. and Dehghan, M. (2014) A Method for Obtaining the Operational Matrix of Fractional Jacobi Functions and Applications. Journal of Vibration and Control, 20, 736-748. http://dx.doi.org/10.1177/1077546312467049

[17] Dhabale, A.S., Dive, R., Aware, M.V. and Das, S. (2015) A New Method for Getting Rational Approximation for Fractional Order Differ Integrals. Asian Journal of Control, 17, 2143-2152.

[18] Zhao, L. and Deng, W. (2014) Jacobian-Predictor-Corrector Approach for Fractional Differential Equations. Advances in Computational Mathematics, 40, 137-165. http://dx.doi.org/10.1007/s10444-013-9302-7

[19] Momani, S. and Odibat, Z. (2006) Analytical Solution of a Time-Fractional Navier-Stokes Equation by Adomian Decomposition Method. Applied Mathematics and Computation, 177, 488-494. http://dx.doi.org/10.1016/j.amc.2005.11.025

[20] Odibat, Z.M. and Momani, S. (2006) Application of Variational Iteration Method to Nonlinear Differential Equations of Fractional Order. International Journal of Nonlinear Sciences and Numerical Simulation, 7, 27-34. http://dx.doi.org/10.1515/IJNSNS.2006.7.1.27

[21] Blank, L. (1996) Numerical Treatment of Differential Equations of Fractional Order. Department of Mathematics, University of Manchester.

[22] Podlubny, I. (2000) Matrix Approach to Discrete Fractional Calculus. Fractional Calculus and Applied Analysis, 3, 359-386.

[23] Podlubny, I., Chechkin, A., Skovranek, T., Chen, Y. and Jara, B.M.V. (2009) Matrix Approach to Discrete Fractional Calculus II: Partial Fractional Differential Equations. Journal of Computational Physics, 228, 3137-3153. http://dx.doi.org/10.1016/j.jcp.2009.01.014

[24] Badakhshan, K.P. and Kamyad, A.V. (2007) Using AVK Method to Solve Nonlinear Problems with Uncertain Para- 
meters. Applied Mathematics and Computation, 189, 27-34. http://dx.doi.org/10.1016/j.amc.2006.11.172

[25] Ding, Y. and Ye, H. (2009) A Fractional-Order Differential Equation Model of HIV Infection of CD4 ${ }^{+}$T-Cells. Mathematical and Computer Modelling, 50, 386-392. http://dx.doi.org/10.1016/j.mcm.2009.04.019

[26] Podlubny, I. (1999) Fractional Differential Equations. Academic Press, San Diego.

[27] Badakhshan, K.P. and Kamyad, A.V. (2007) Numerical Solution of Nonlinear Optimal Control Problems Using Nonlinear Programming. Applied Mathematics and Computation, 187, 1511-1519. http://dx.doi.org/10.1016/j.amc.2006.09.074

[28] Zeid, S.S. and Kamyad, A.V. (2014) On Generalized High Order Derivatives of Nonsmooth Functions. American Journal of Computational Mathematics, 4, 317-328. http://dx.doi.org/10.4236/ajcm.2014.44028

[29] Odibat, Z. and Momani, S. (2008) An Algorithm for the Numerical Solution of Differential Equations of Fractional Order. Journal of Applied Mathematics \& Informatics, 26, 15-27. 